\title{
SOME RESULTS ON CONTROLLED $K$-FRAMES IN HILBERT SPACES
}

\author{
M. NOURI ${ }^{1,2, *}$, A. RAHIMI ${ }^{2}$ AND SH. NAJAFZADEH ${ }^{2}$ \\ ${ }^{1}$ Department of Mathematics, Payame Noor University, P.O.Box 19395-3697 Tehran, Iran \\ ${ }^{2}$ Department of Mathematics, University of Maragheh, Maragheh, Iran \\ *Corresponding author: mohammadnoori562@yahoo.com

\begin{abstract}
Controlled frames have been introduced to improve the numerical efficiency of iterative algorithms for inverting the frame operator. Also $K$-frames have been introduced to study atomic systems with respect to bounded linear operator. In this paper, the notion of controlled $K$-frames will be studied and it will be shown that controlled $K$-frames are equivalent to $K$-frames under some mild conditions. Finally, the stability of controlled $K$-Bessel sequences under perturbation will be discussed with some examples.
\end{abstract}

\section{INTRODUCTION}

Frames in Hilbert spaces were first proposed by Duffin and Schaeffer to deal with nonharmonic Fourier series in 1952 [9] and widely studied from 1986 by Daubechies et al. [10]. Now, frames play an important role not only in the theoretics also in many kinds of applications and have been widely applied in signal processing [13], sampling [11,12], coding and communications [19], filter bank theory [3], system modeling [8] and so on.

Over the years, various extentions of the frame theory have been investigated and proposed, such as the fusion frames $[5,6]$ to deal with hierarchical data processing, $g$-frames [20] by Sun to deal with all existing frames as united object, oblique dual frames [11] by Elder to deal with sampling reconstructions, and etc.

Received $11^{\text {th }}$ September, 2017; accepted $29^{\text {th }}$ November, 2017; published $3^{\text {rd }}$ January, 2018.

2000 Mathematics Subject Classification. Primary 42C40; Secondary 41A58, 47A58.

Key words and phrases. Bessel sequence; controlled frame; frame; $K$-frame; perturbation.

(C) 2018 Authors retain the copyrights of their papers, and all open access articles are distributed under the terms of the Creative Commons Attribution License. 
The notion of $K$-frames were recently introduced by L. Găvruta to study the atomic systems with respect to a bounded linear operator $K$ in Hilbert spaces. $K$-frames are more general than ordinary frames in sense that the lower frame bound only holds for the elements in the range of the $K$, where $K$ is a bounded linear operator in a separable Hilbert Space $H$.

Recent addition to these generalized frames are the controlled frames [1]. Controlled frames have been introduced to improve the numerical efficiency of iterative algorithms for inverting the frame operator on abstract Hilbert spaces, however, they were used earlier just as a tool for spherical wavelets [2]. The main advantage of these frames lies in the fact that they retain all the advantages of standard frames but additionally they give a generalized way to check the frame condition while offering a numerical advantage in the sense of preconditioning. Recent developments in this direction can be found in [14-18] and the references therein.

In this paper, the concept of controlled $K$-frame will be defined and it will be shown that any controlled $K$-frame is equivalent to a $K$-frame. Finally, we will discuss the stability of controlled $K$-Bessel sequences under perturbation.

Throughout this paper, $H$ is a separable Hilbert space, $B(H)$ is the family of all bounded linear operators on $H$ and $K \in B(H) . G L(H)$ denotes the set of all bounded linear operators which have bounded inverses and $G L^{+}(H)$ denotes the set of all positive operators in $G L(H)$.

The paper is organized as follows:

Section 2 contains some preliminary result. In section 3, we define the concept of controlled $K$-frame and we will show that controlled $K$-frames are equivalent to $K$-frames. In section 4 , we discuss the stability of a more general perturbation for controlled $K$-Bessel sequence. In section 5 , we will examine with some examples the perturbation of controlled $K$-Bessel sequences.

\section{Preliminaries and notations}

In this section, some necessary definitions and theorems are presented.

Definition 2.1. A bounded operator $T \in B(H)$ is called positive (respectively, non-negative), if $\langle T f, f\rangle>0$ for all $f \neq 0$ (respectively, $\langle T f, f\rangle \geq 0$ for all $f$ ).

Every non-negative operator is clearly self-adjoint.

If $A \in B(H)$ is non-negative, then there exists a unique non-negative operator $B$ such that $B^{2}=A$. Furthermore, $B$ commutes with every operator that commutes with $A$. This will be denoted by $B=A^{\frac{1}{2}}$.

Let $B^{+}(H)$ be the set of positive operators on $H$. For self-adjoint operators $T_{1}$ and $T_{2}$, the notation $T_{1} \leq T_{2}$ or $T_{2}-T_{1} \geq 0$ means

$$
\left\langle T_{1} f, f\right\rangle \leq\left\langle T_{2} f, f\right\rangle \quad, \forall f \in H
$$


The following result is needed in the sequel, but straightforward to prove:

Proposition 2.1. [1] Let $T: H \rightarrow H$ be a linear operator. Then the following conditions are equivalent:

a. There exist $m>0$ and $M<\infty$, such that $m I \leq T \leq M I$,

b. $T$ is positive and there exist $m>0$ and $M<\infty$, such that $m\|f\|^{2} \leq\left\|T^{\frac{1}{2}} f\right\|^{2} \leq M\|f\|^{2}$ for all $f \in H$,

c. $T$ is positive and $T^{\frac{1}{2}} \in G L(H)$,

d. There exists a self-adjoint operator $A \in G L(H)$, such that

$$
A^{2}=T
$$

e. $T \in G L^{+}(H)$,

f. There exist constants $m>0$ and $M<\infty$ and operator

$C \in G L^{+}(H)$, such that $m^{\prime} C \leq T \leq M^{\prime} C$,

g. For every $C \in G L^{+}(H)$, there exist constants $m>0$ and

$M<\infty$, such that $m^{\prime} C \leq T \leq M^{\prime} C$.

It is well-known that all bounded operators $U$ on a Hilbert space $H$ are not invertible: an operator $U$ needs to be injective and surjective in order to be invertible. For doing this, one can use right-inverse operator. The following lemma shows that if an operator $U$ has closed range, there exists a right-inverse operator $U^{\dagger}$ in the following sense:

Lemma 2.1. [7] Let $H_{1}$ and $H_{2}$ be Hilbert spaces and suppose that $U: H_{2} \rightarrow H_{1}$ is a bounded operator with closed range $R_{U}$. Then there exists a bounded operator $U^{\dagger}: H_{1} \rightarrow H_{2}$ which

$$
U U^{\dagger} x=x, \quad \forall x \in R_{U}
$$

The operator $U^{\dagger}$ in the Lemma 2.3 is called the pseudo-inverse of $U$.

In the literature, one will often see the pseudo-inverse of an operator $U$ with closed range defined as the unique operator $U^{\dagger}$ satisfying that

$$
N_{U^{\dagger}}=R_{U}^{\perp}, \quad R_{U^{\dagger}}=N_{U}^{\perp}, \quad U U^{\dagger} x=x, \quad \forall x \in R_{U} .
$$

Definition 2.2. A sequence $\left\{f_{i}\right\}_{i \in I}$ in $H$ is called a frame for $H$, if there exist constants $0<A \leq B<\infty$ such that

$$
A\|f\|^{2} \leq \sum_{i \in I}\left|\left\langle f, f_{i}\right\rangle\right|^{2} \leq B\|f\|^{2}, \quad \forall f \in H .
$$

If $A=B$, then $\left\{f_{i}\right\}_{i \in I}$ is called a tight frame and if $A=B=1$, it is called a Parseval frame.

If only the right inequality of the above inequality holds, $\left\{f_{n}\right\}_{n \in I}$ is called a Bessel sequence. 
Remark 2.1. The frame operator $S f=\sum_{i \in I}\left\langle f, f_{i}\right\rangle f_{i}$ associated with a frame $\left\{f_{i}\right\}_{i \in I}$ is a bounded, invertible and positive operator on $H$. This provides the reconstruction formulas

$$
f=S^{-1} S f=\sum_{i \in I}\left\langle f, f_{i}\right\rangle S^{-1} f_{i}=\sum_{i \in I}\left\langle f, S^{-1} f_{i}\right\rangle f_{i}, \quad \forall f \in H .
$$

Furthermore, $A I \leq S \leq B I$ and $B^{-1} I \leq S^{-1} \leq A^{-1} I$.

Definition 2.3. Let $C \in G L(H)$. A frame controlled by the operator $C$ or $C$-controlled frame is a family of vectors $\left\{f_{i}\right\}_{i \in I}$ in $H$, such that there exist constants $0<m_{C} \leq M_{C}<\infty$, verifying

$$
m_{C}\|f\|^{2} \leq \sum_{i \in I}\left\langle f, f_{i}\right\rangle\left\langle C f_{i}, f\right\rangle \leq M_{C}\|f\|^{2}, \quad \forall f \in H .
$$

The controlled frame operator $S$ is defined by

$$
S f=\sum_{i \in I}\left\langle f, f_{i}\right\rangle C f_{i}, \quad \forall f \in H
$$

Definition 2.4. Let $K \in B(H)$. A sequence $\left\{f_{n}\right\}_{n=1}^{\infty} \subset H$ is called a $K$-frame for $H$, if there exist constants $A, B>0$ such that

$$
A\left\|K^{*} f\right\|^{2} \leq \sum_{n=1}^{\infty}\left|\left\langle f, f_{n}\right\rangle\right|^{2} \leq B\|f\|^{2}, \quad \forall f \in H
$$

We call $A$ and $B$ the lower and upper frame bounds for $K$-frame, respectively.

If only the right inequality of the above inequality holds, $\left\{f_{n}\right\}_{n=1}^{\infty} \subset H$ is called a K-Bessel sequence.

Remark 2.2. If $K=I$, then $K$-frame are just the ordinary frame.

Remark 2.3. In the following, we will assume that $R(K)$ is closed, since this can assume that the pseudoinverse $K^{\dagger}$ of $K$ exists.

Because of the higher generality of $K$-frames, some properties of ordinary frames can not hold for $K$ frames, such as the frame operator of a $K$-frame is not an isomorphism. For more differences between $K$-frames and ordinary frames, we refer to [21].

Definition 2.5. Let $K \in B(H)$. A sequence $\left\{f_{n}\right\}_{n=1}^{\infty} \subset H$ is called an atomic system for $K$, if the following conditions are satisfied:

(1) $\left\{f_{n}\right\}_{n=1}^{\infty}$ is a Bessel sequence.

(2) For any $x \in H$, there exists $a_{x}=\left\{a_{n}\right\} \in l^{2}$ such that

$$
K x=\sum_{n=1}^{\infty} a_{n} f_{n}
$$

where $\left\|a_{x}\right\|_{l^{2}} \leq C\|x\|, C$ is positive constant. 
Suppose that $\left\{f_{n}\right\}_{n=1}^{\infty}$ is a $K$-frame for $H$. Obviously it is a Bessel sequence, so we can define the following operator

$$
T: l^{2} \rightarrow H, \quad T a=\sum_{n=1}^{\infty} a_{n} f_{n}, \quad a=\left\{a_{n}\right\} \in l^{2},
$$

it follows that

$$
\begin{gathered}
T^{*}: H \rightarrow l^{2} \\
T^{*} f=\left\{\left\langle f, f_{n}\right\rangle\right\}_{n=1}^{\infty}, \quad \forall f \in H .
\end{gathered}
$$

Let $S=T T^{*}$, we obtain

$$
S f=\sum_{n=1}^{\infty}\left\langle f, f_{n}\right\rangle f_{n}, \quad \forall f \in H .
$$

we call $T, T^{*}$ and $S$ the synthesis operator, analysis operator and frame operator for $K$-frame $\left\{f_{n}\right\}_{n=1}^{\infty}$, respectively.

The following theorem gives a characterization of $K$-frames in Hilbert spaces.

Proposition 2.2. Let $\left\{f_{n}\right\}_{n=1}^{\infty}$ be a Bessel sequence in $H$. Then $\left\{f_{n}\right\}_{n=1}^{\infty}$ is a $K$-frame for $H$, if and only if there exists $A>0$ such that

$$
S \geq A K K^{*}
$$

where $S$ is the frame operator for $\left\{f_{n}\right\}_{n=1}^{\infty}$.

Proof. The sequence $\left\{f_{n}\right\}_{n=1}^{\infty}$ is a $K$-frame for $H$ with frame bounds $A, B$ and frame operator $S$ if and only if

$$
A\left\|K^{*} f\right\|^{2} \leq \sum_{K=1}^{\infty}\left|\left\langle f, f_{n}\right\rangle\right|^{2}=\langle S f, f\rangle \leq B\|f\|^{2}, \forall f \in H
$$

that is

$$
\left\langle A K K^{*} f, f\right\rangle \leq\langle S f, f\rangle \leq\langle B f, f\rangle, \forall f \in H .
$$

so the conclusion holds.

Remark 2.4. Frame operator of a $K$-frames is not invertible on $H$ in general, but we can show that it is invertible on the subspace $R(K) \subset H$. In fact, since $R(K)$ is closed, there exists a pseudo-inverse $K^{\dagger}$ of $K$, such that $K K^{\dagger} f=f, \forall f \in R(K)$, namely $\left.K K^{\dagger}\right|_{R(K)}=I_{R(K)}$, so we have $I_{R(K)}^{*}=\left(\left.K^{\dagger}\right|_{R(K)}\right)^{*} K^{*}$. Hence for any $f \in R(K)$, we obtain

$$
\|f\|=\left\|\left(\left.K^{\dagger}\right|_{R(K)}\right)^{*} K^{*} f\right\| \leq\left\|K^{\dagger}\right\| \cdot\left\|K^{*} f\right\|,
$$

that is, $\left\|K^{*} f\right\|^{2} \geq\left\|K^{\dagger}\right\|^{-2}\|f\|^{2}$. Combined with (2.2), we have

$$
\langle S f, f\rangle \geq A\left\|K^{*} f\right\|^{2} \geq A\left\|K^{\dagger}\right\|^{-2}\|f\|^{2}, \forall f \in R(K) .
$$


So, from the definition of $K$-frame we have

$$
A\left\|K^{\dagger}\right\|^{-2}\|f\| \leq\|S f\| \leq B\|f\|, \forall f \in R(K)
$$

which implies that $S: R(K) \rightarrow S(R(K))$ is a homeomorphism. Furthermore, we have

$$
B^{-1}\|f\| \leq\left\|S^{-1} f\right\| \leq A^{-1}\left\|K^{\dagger}\right\|^{2}\|f\|, \forall f \in S(R(K)) .
$$

\section{Controlled $K$-Frames}

Controlled frames for spherical wavelets were introduced in [2] to get a numerically more efficient approximation algorithm and the related theory. For general frames, it was developed in [1]. For getting a numerical solution of a linear system of equations $A x=b$, we can solve the system of equations $P A x=P b$, where $P$ is a suitable preconditioning matrix. It was the main motivation for introducing controlled frames in [2]. Controlled frames extended to $g$-frames in [17] and for fusion frames in [15]. In this section, the concept of controlled frames and controlled Bessel sequences will be extended to $K$-frames and it will be shown that controlled $K$-frames are equivalent $K$-frames.

Definition 3.1. Let $C \in G L^{+}(H)$ and let $C K=K C$. The family $\left\{f_{n}\right\}_{n=1}^{\infty}$ is called $C$-controlled $K$-frame for $H$, if $\left\{f_{n}\right\}_{n=1}^{\infty}$ is a $K$-Bessel sequence and there exist constants $A>0$ and $B<\infty$ such that

$$
A\left\|C^{\frac{1}{2}} K^{*} f\right\|^{2} \leq \sum_{n=1}^{\infty}\left\langle f, f_{n}\right\rangle\left\langle f, C f_{n}\right\rangle \leq B\|f\|^{2}, \forall f \in H .
$$

The constants $A$ and $B$ are called $C$-controlled $K$-frame bounds. If $C=I$, the $C$-controlled $K$-frame $\left\{f_{n}\right\}_{n=1}^{\infty}$ is a $K$-frame for $H$ with bounds $A$ and $B$.

If the second part of the above inequality holds, it called $C$-controlled $K$-Bessel sequence with bound B.

Definition 3.2. Let $C \in G L^{+}(H)$. A sequence $\left\{f_{n}\right\}_{n=1}^{\infty} \in H$ is a $C$-controlled Bessel sequence for $H$ if and only if the operator

$$
L_{C}: H \rightarrow H, L_{C} f=\sum_{n=1}^{\infty}\left\langle f, f_{n}\right\rangle C f_{n}, \quad \forall f \in H .
$$

is well defined and there exists constant $B<\infty$ such that

$$
\sum_{n=1}^{\infty}\left\langle f, f_{n}\right\rangle\left\langle f, C f_{n}\right\rangle \leq B\|f\|^{2}, \forall f \in H
$$

Definition 3.3. The operator $L_{C}: H \rightarrow H$ and $L_{C} f=\sum_{n=1}^{\infty}\left\langle f, f_{n}\right\rangle C f_{n}$ where $f \in H$ is called the $C$-controlled Bessel sequence operator, also $L_{C} f=C S f$.

The following lemma characterizes $C$-controlled $K$-frames in term of their operators.

Lemma 3.1. Let $\left\{f_{n}\right\}_{n=1}^{\infty}$ be a C-controlled $K$-frame in $H$, for $C \in G L^{+}(H)$. Then

$$
A I\left\|C^{\frac{1}{2}} K^{\dagger}\right\|^{2} \leq L_{C} \leq B I
$$


Proof. Suppose that $\left\{f_{n}\right\}_{n=1}^{\infty}$ is a $C$-controlled $K$-frame with bounds $A$ and $B$. Then

$$
A\left\|C^{\frac{1}{2}} K^{*} f\right\|^{2} \leq \sum_{n=1}^{\infty}\left\langle f, f_{n}\right\rangle\left\langle f, C f_{n}\right\rangle \leq B\|f\|^{2}, \forall f \in H .
$$

For $f \in H$

$$
A\left\|C^{\frac{1}{2}} K^{*} f\right\|^{2} \leq\left\langle f, L_{C} f\right\rangle \leq B\|f\|^{2}
$$

i.e.

$$
A\left\|C^{\frac{1}{2}} K^{*}\right\|^{2} I \leq L_{C} \leq B I .
$$

The following proposition shows that for evaluation a family $\left\{f_{n}\right\}_{n=1}^{\infty} \subset H$ to be a controlled $K$-frame it is sufficient to check just a simple operator inequality.

Proposition 3.1. Let $\left\{f_{n}\right\}_{n=1}^{\infty}$ be a Bessel sequence in $H$ and $C \in G L^{+}(H)$. Then $\left\{f_{n}\right\}_{n=1}^{\infty}$ is a C-controlled $K$-frame for $H$ if and only if there exists $A>0$ such that $C S \geq C A K K^{*}$.

Proof. The sequence $\left\{f_{n}\right\}_{n=1}^{\infty}$ is a controlled $K$-frame for $H$ with frame bounds $A, B$ and frame operator $S$ if and only if

$$
A\left\|C^{\frac{1}{2}} K^{*} f\right\|^{2} \leq \sum_{n=1}^{\infty}\left\langle f, f_{n}\right\rangle\left\langle f, C f_{n}\right\rangle \leq B\|f\|^{2}, \forall f \in H
$$

That is,

$$
\left\langle C A K K^{*} f, f\right\rangle \leq\langle C S f, f\rangle \leq\langle B f, f\rangle, \forall f \in H
$$

The following proposition shows that any controlled $K$-frame is a $K$-frame.

Proposition 3.2. Let $\left\{f_{n}\right\}_{n=1}^{\infty}$ be a $C$-controlled $K$-frame and $C \in G L^{+}(H)$. Then $\left\{f_{n}\right\}_{n=1}^{\infty}$ is a K-frame for $H$.

Proof. Suppose that $\left\{f_{n}\right\}_{n=1}^{\infty}$ is a controlled $K$-frame with bounds $A$ and $B$. Then for any $f \in H$,

$$
\begin{aligned}
A\left\|K^{*} f\right\|^{2} & =A\left\|C^{-\frac{1}{2}} C^{\frac{1}{2}} K^{*} f\right\|^{2} \\
& \leq A\left\|C^{\frac{1}{2}}\right\|^{2}\left\|C^{-\frac{1}{2}} K^{*} f\right\|^{2} \\
& \leq\left\|C^{\frac{1}{2}}\right\|^{2} \sum_{n=1}^{\infty}\left\langle f, f_{n}\right\rangle\left\langle f, C^{0} f_{n}\right\rangle \\
& =\left\|C^{\frac{1}{2}}\right\|^{2} \sum_{n=1}^{\infty}\left|\left\langle f, f_{n}\right\rangle\right|^{2} .
\end{aligned}
$$

Hence for $f \in H$,

$$
A\left\|C^{\frac{1}{2}}\right\|^{-2}\left\|K^{*} f\right\|^{2} \leq \sum_{n=1}^{\infty}\left|\left\langle f, f_{n}\right\rangle\right|^{2}
$$


On the other hand for every $f \in H$,

$$
\begin{aligned}
\sum_{n=1}^{\infty}\left|\left\langle f, f_{n}\right\rangle\right|^{2} & =\langle f, S f\rangle \\
& =\left\langle f, C^{-1} C S f\right\rangle \\
& =\left\langle\left(C^{-1} C S\right)^{\frac{1}{2}} f,\left(C^{-1} C S\right)^{\frac{1}{2}} f\right\rangle \\
& =\left\|\left(C^{-1} C S\right)^{\frac{1}{2}} f\right\|^{2} \\
& \leq\left\|C^{-\frac{1}{2}}\right\|^{2}\left\|(C S)^{\frac{1}{2}} f\right\|^{2} \\
& =\left\|C^{-\frac{1}{2}}\right\|^{2}\langle f, C S f\rangle \\
& \leq\left\|C^{-\frac{1}{2}}\right\|^{2} B\|f\|^{2} .
\end{aligned}
$$

These inequalities yields that $\left\{f_{n}\right\}_{n=1}^{\infty}$ is a $K$-frame with bounds $A\left\|C^{\frac{1}{2}}\right\|^{-2}$ and $B\left\|C^{-\frac{1}{2}}\right\|^{2}$.

The following proposition show that any $K$-frame is a controlled $K$-frame under some conditions.

Proposition 3.3. Let $C \in G L^{+}(H)$ be a self adjoint and $K C=C K$, if $\left\{f_{n}\right\}_{n=1}^{\infty}$ is $K$-Frame for $H$, then $\left\{f_{n}\right\}_{n=1}^{\infty}$ is a $C$-controlled $K$-frame for $H$.

Proof. Suppose that $\left\{f_{n}\right\}_{n=1}^{\infty}$ be a $K$-frame with bounds $A^{\prime}$ and $B^{\prime}$. Then for all $f \in H$,

$$
\begin{aligned}
& A^{\prime}\left\|K^{*} f\right\|^{2} \leq \sum_{n=1}^{\infty}\left|\left\langle f, f_{n}\right\rangle\right|^{2} \leq B^{\prime}\|f\|^{2} . \\
& A^{\prime}\left\|C^{\frac{1}{2}} K^{*} f\right\|^{2}=A^{\prime}\left\|K^{*} C^{\frac{1}{2}} f\right\|^{2} \leq \sum_{n=1}^{\infty}\left\langle C^{\frac{1}{2}} f, f_{n}\right\rangle\left\langle C^{\frac{1}{2}} f, f_{n}\right\rangle \\
&=\left\langle C^{\frac{1}{2}} f, \sum_{n=1}^{\infty}\left\langle f_{n}, C^{\frac{1}{2}} f\right\rangle f_{n}\right\rangle \\
&=\left\langle C^{\frac{1}{2}} f, C^{\frac{1}{2}} S f\right\rangle=\langle f, C S f\rangle .
\end{aligned}
$$

Hence $A^{\prime}\left\|C^{\frac{1}{2}} K^{*} f\right\|^{2} \leq\langle f, C S f\rangle$ for every $f \in H$. On the other hand for every $f \in H$,

$$
|\langle f, C S f\rangle|^{2}=\left|\left\langle C^{*} f, S f\right\rangle\right|^{2}=|\langle C f, S f\rangle|^{2} \leq\|C f\|^{2}\|S f\|^{2} \leq\|C\|^{2}\|f\|^{2} B\|f\|^{2} .
$$

Hence,

$$
A^{\prime}\left\|C^{\frac{1}{2}} K^{*} f\right\|^{2} \leq\langle f, C S f\rangle \leq B^{\prime}\|C\|\|f\|^{2} .
$$

Therefore $\left\{f_{n}\right\}_{n=1}^{\infty}$ is a $C$-controlled $K$-frame with bounds $A^{\prime}$ and $B^{\prime}\|C\|$. 


\section{Perturbation for Controlled $K$-Bessel Sequences}

One of the most important problems in the studying of frames and its applications specially on wavelet and Gabor systems is the invariance of these systems under perturbation. At the first, the problem of perturbation studied by Paley and Wiener for bases and then extended to frames. There are many versions of perturbation of frames in Hilbert spaces, Banach space, Hilbert $C^{*}$-modules and etc. In the last decade, several authors have generalized the Paley-Wiener perturbation theorem to the perturbation of frames in Hilbert spaces. The most general result of these was the following obtained by Casazza and Christensen [4].

In this section, we mainly give an important on stability of perturbation for $K$-frames.To do this, we have to introduce tree lemmas below first.

Lemma 4.1. [7] A sequence $\left\{f_{n}\right\}_{n=1}^{\infty} \subset H$ is a Bessel sequence with bound $B$ in $H$, if and only if the operator

$T: L^{2} \rightarrow H, T a=\sum a_{n} f_{n}$ is well-defined and bounded operator with $\|T\| \leq \sqrt{B}$.

Lemma 4.2. [Y] If $\left\{f_{n}\right\}_{n=1}^{\infty}$ is an ordinary frame for $H$, then $\left\{K f_{n}\right\}_{n=1}^{\infty}$ is a K-Frames for $H$.

Lemma 4.3. [7] Let $T_{1} \in L(X, Y)$ and let $T_{2}: X \rightarrow Y$ be linear. If there exist two constants $\lambda_{1}, \lambda_{2} \in[0,1]$ such that

$$
\left\|T_{1} x-T_{2} x\right\| \leq \lambda_{1}\left\|T_{1} x\right\|+\lambda_{2}\left\|T_{2} x\right\|, \forall x \in X
$$

then $T_{2} \in L(X, Y)$. Moreover, if $T_{1}$ is invertible on $X$, then $T_{2}$ is also invertible on $X$, and we have

$$
\frac{1-\lambda_{1}}{1+\lambda_{2}}\left\|T_{1} x\right\| \leq\left\|T_{2} x\right\| \leq \frac{1+\lambda_{1}}{1-\lambda_{2}}\left\|T_{1} x\right\|, \forall x \in X
$$

and

$$
\frac{1-\lambda_{2}}{1+\lambda_{1}} \cdot \frac{1}{\left\|T_{1}\right\|}\|y\| \leq\left\|T_{2}^{-1} y\right\| \leq \frac{1+\lambda_{2}}{1-\lambda_{1}}\left\|T_{1}^{-1}\right\|\|y\|, \forall y \in Y
$$

Theorem 4.1. [4] Let $\left\{x_{j}\right\}_{j \in J}$ be a frame for a Hilbert space $H$ with frame bounds $C$ and D. Assume that $\left\{y_{j}\right\}_{j \in J}$ is a sequence of $H$ and that there exist $\lambda_{1}, \lambda_{2}, \mu>0$ such that max $\left\{\lambda_{1}+\frac{\mu}{\sqrt{C}}, \lambda_{2}\right\}<1$. Suppose one of the following conditions holds for any finite scalar sequence $\left\{c_{j}\right\}$ and every $x \in H$. Then $\left\{y_{j}\right\}_{j \in J}$ is also a frame for $H$;

(1) $\left(\sum_{j \in J}\left|\left\langle x, x_{j}-y_{j}\right\rangle\right|^{2}\right)^{\frac{1}{2}} \leq \lambda_{1}\left(\sum_{j \in J}\left|\left\langle x, x_{j}\right\rangle\right|^{2}\right)^{\frac{1}{2}}+\lambda_{2}\left(\sum_{j \in J}\left|\left\langle x, y_{j}\right\rangle\right|^{2}\right)^{\frac{1}{2}}+\mu\|x\|$

(2) $\left\|\sum_{i=1}^{n} c_{j}\left(x_{j}-y_{j}\right)\right\| \leq \lambda_{1}\left\|\sum_{i=1}^{n} c_{j} x_{j}\right\|+\lambda_{2}\left\|\sum_{i=1}^{n} c_{j} y_{j}\right\|+\mu\left(\sum_{i=1}^{n}\left|c_{j}\right|^{2}\right)^{\frac{1}{2}}$.

Moreover, if $\left\{x_{j}\right\}_{j \in J}$ is a Riesz basis for $H$ and $\left\{y_{j}\right\}_{j \in J}$ satisfies $(2)$, then $\left\{y_{j}\right\}_{j \in J}$ is also a Riesz basis for $H$.

The perturbation theorem investigated by X. Xiao, Y. Zhu, L. Găvruta to K-frames [21]: 
Theorem 4.2. Suppose that $\left\{f_{n}\right\}_{n=1}^{\infty}$ is a $K$-frame for $H$, and $\alpha, \beta \in[0, \infty]$, such that $\max \left\{\alpha+\gamma \sqrt{A^{-1}}\left\|K^{+}\right\|, \beta\right\}<$ 1.

If $\left\{g_{n}\right\}_{n=1}^{\infty} \subset H$ and satisfy,

$$
\left\|\sum_{k=1}^{n} c_{k}\left(f_{k}-g_{k}\right)\right\| \leq \alpha\left\|\sum_{k=1}^{n} c_{k} f_{k}\right\|+\beta\left\|\sum_{k=1}^{n} c_{k} g_{k}\right\|+\gamma\left(\sum_{k=1}^{n}\left|c_{k}\right|^{2}\right)^{\frac{1}{2}}
$$

for any $c_{i}(i \in \mathbb{N})$, then $\left\{g_{n}\right\}_{n=1}^{\infty}$ is a $P_{Q(R(K))} K$-Frame for $H$, with frame bounds

$$
\frac{\left[\sqrt{A}\left\|K^{+}\right\|^{-1}(1-\alpha)-\gamma\right]^{2}}{(1+\beta)^{2}\|K\|^{2}}, \frac{[\sqrt{B}(1+\alpha)+\gamma]^{2}}{(1-\beta)^{2}},
$$

where $P_{Q(R(K))}$ is a orthogonal projection operator for $H$ to $Q(R(K)), Q=U T^{*}, T, U$ are synthesis operator for $\left\{f_{n}\right\}_{n=1}^{\infty}$ and $\left\{g_{n}\right\}_{n=1}^{\infty}$ respectively.

Motivating the above theorems, we prove perturbation for controlled $K$-Bessel sequences.

Theorem 4.3. Suppose that $\left\{f_{n}\right\}_{n=1}^{+\infty} \subset H$ is a $C$-controlled $K$-frame for $H$, with frame bounds $A$, B, and $\alpha, \beta, \gamma \in[0, \infty)$, such that

$$
\operatorname{Max}\left\{\alpha+\gamma \sqrt{A^{-1}}\left\|K^{+}\right\|, \beta\right\}<1
$$

If $\left\{g_{n}\right\}_{n=1}^{+\infty} \subset H$ and satisfy,

$$
\begin{aligned}
\left\|\sum_{k=1}^{n} c_{k} f_{k}-\sum_{k=1}^{n} c_{k} g_{k}\right\| \leq & \alpha\|c\|\left\|\sum_{k=1}^{n} c_{k} f_{k}\right\|+\beta\left\|\sum_{k=1}^{n} c_{k} g_{k}\right\| \\
& +\gamma\left(\sum_{k=1}^{n}\left|c_{k}\right|^{2}\right)^{\frac{1}{2}},
\end{aligned}
$$

for any $c_{i}$, then $\left\{g_{n}\right\}_{n=1}^{+\infty}$ is a controlled $K$-Bessel sequence for $H$ with bound $\left(\frac{(1+\alpha\|c\|) \sqrt{B}+\gamma}{1-\beta}\right)^{2}$, where $T, U$ are the synthesis operator for $\left\{f_{n}\right\}_{n=1}^{+\infty}$ and $\left\{g_{n}\right\}_{n=1}^{+\infty}$, respectively.

Proof. Let $\left\{f_{n}\right\}_{n=1}^{+\infty}$ be a frame for H, so by lemma 4.1, the frame operator $T$ is bounded and $\|T\| \leq \sqrt{B}$.

The condition (4.1) implies that for all finite sequences $\left\{c_{k}\right\}$,

$$
\begin{aligned}
\left\|\sum_{k=1}^{n} c_{k} g_{k}\right\|= & \left\|-\sum_{k=1}^{n} c_{k}\left(f_{k}-g_{k}\right)+\sum_{k=1}^{n} c_{k} f_{k}\right\| \leq\left\|-\sum_{k=1}^{n} c_{k}\left(f_{k}-g_{k}\right)\right\|+\left\|\sum_{k=1}^{n} c_{k} f_{k}\right\| \\
& \leq(1+\alpha\|c\|)\left\|\sum_{k=1}^{n} c_{k} f_{k}\right\|+\beta\left\|\sum_{k=1}^{n} c_{k} g_{k}\right\|+\gamma\left(\sum_{k=1}^{n}\left|c_{k}\right|^{2}\right)^{\frac{1}{2}} .
\end{aligned}
$$

This calculation actually holds for all $\left\{c_{k}\right\}_{k=1}^{+\infty} \in l^{2}(\mathbb{N})$. To see this, at the first we have to prove that $\sum_{k=1}^{\infty} c_{k} g_{k}$ is convergent for any given $\left\{c_{k}\right\}_{k=1}^{+\infty} \in l^{2}(\mathbb{N})$.

Given $n, m \in \mathbb{N}$ with $n>m$,

$$
\left\|\sum_{k=1}^{n} c_{k} f_{k}-\sum_{k=1}^{m} c_{k} f_{k}\right\|=\left\|\sum_{k=m+1}^{n} c_{k} f_{k}\right\|
$$




$$
\leq(1+\alpha\|c\|)\left\|\sum_{k=m+1}^{n} c_{k} f_{k}\right\|+\beta\left\|\sum_{k=m+1}^{n} c_{k} g_{k}\right\|+\gamma\left(\sum_{k=m+1}^{n}\left|c_{k}\right|^{2}\right)^{\frac{1}{2}} .
$$

Since $\left\{c_{k}\right\}_{k=1}^{\infty} \in l^{2}(\mathbb{N})$ and $\sum_{k=1}^{\infty} c_{k} f_{k}$ is convergent, this implies that $\left\{\sum_{k=1}^{n} c_{k} f_{k}\right\}_{n=1}^{\infty}$ is a Cauchy sequence in $H$ and therefore convergent, thus the pre-frame operator $U$ is well defined on $l^{2}(\mathbb{N})$; it follows that for all $\left\{c_{k}\right\}_{k=1}^{\infty} \in l^{2}(\mathbb{N})$,

$$
\left\|\sum_{k=1}^{\infty} c_{k} g_{k}\right\| \leq(1+\alpha\|c\|)\left\|\sum_{k=1}^{\infty} c_{k} f_{k}\right\|+\beta\left\|\sum_{k=1}^{\infty} c_{k} g_{k}\right\|+\gamma\left(\sum_{k=1}^{\infty}\left|c_{k}\right|^{2}\right)^{\frac{1}{2}},
$$

In terms of the operator $T, U,(4.3)$ states that

$$
\begin{aligned}
& \left\|U\left\{c_{k}\right\}_{k=1}^{\infty}\right\| \leq(1+\alpha\|c\|)\left\|T\left\{c_{k}\right\}_{k=1}^{\infty}\right\|+\beta\left\|U\left\{c_{k}\right\}_{k=1}^{\infty}\right\|+\gamma\left(\sum_{k=1}^{\infty}\left|c_{k}\right|^{2}\right)^{\frac{1}{2}} \\
& \leq((1+\alpha\|c\|) \sqrt{B}+\gamma)\left(\sum_{k=1}^{\infty}\left|c_{k}\right|^{2}\right)^{\frac{1}{2}}+\beta\left\|U\left\{c_{k}\right\}_{k=1}^{\infty}\right\|, \forall\left\{c_{k}\right\}_{k=1}^{\infty} \in l^{2}(\mathbb{N}) .
\end{aligned}
$$

So

$$
\left\|U\left\{c_{k}\right\}_{k=1}^{\infty}\right\| \leq \frac{((1+\alpha\|c\|) \sqrt{B}+\gamma)}{1-\beta}\left(\sum_{k=1}^{\infty}\left|c_{k}\right|^{2}\right)^{\frac{1}{2}}
$$

Via lemma 4.1, this estimation shows that $\left\{g_{k}\right\}_{k=1}^{\infty}$ is a Bessel sequence with bound $\frac{((1+\alpha\|c\|) \sqrt{B}+\gamma)^{2}}{(1-\beta)^{2}}$.

\section{Examples}

In this section, we give some examples that examines the stability of the controlled $K$-Bessel sequences under perturbation.

Example 5.1. Suppose that $H=\mathbb{C}^{3},\left\{g_{n}\right\}_{n=1}^{3}=\left\{e_{1}, e_{2}, e_{3}\right\}$, where

$$
e_{1}=\left(\begin{array}{l}
1 \\
0 \\
0
\end{array}\right), \quad e_{2}=\left(\begin{array}{l}
0 \\
1 \\
0
\end{array}\right), \quad e_{3}=\left(\begin{array}{l}
0 \\
0 \\
1
\end{array}\right) \text {. }
$$

Now define $K \in L(H)$ as follows

$$
K: H \rightarrow H, \quad K e_{1}=e_{1}, K e_{2}=e_{1}, K e_{3}=e_{2}
$$

Obviously, $\left\{g_{n}\right\}_{n=1}^{3}$ is an ordinary frame for $H$. By Lemma 4.2, we know that $\left\{f_{n}\right\}_{n=1}^{3}=\left\{K g_{n}\right\}_{n=1}^{3}$ is a $K$-frame for $H$. By Proposition 3.3, we can show that $\left\{f_{n}\right\}_{n=1}^{+\infty}$ is a controlled $K$-frame for $H$.

Example 5.2. Let $H=\mathbb{C}^{3},\left\{e_{i}\right\}_{i=1}^{3}$ be the orthonormal basis for $H$. Now define $K \in L(H)$ as follows

$$
K: H \rightarrow H, \quad K e_{1}=2 e_{1}, K e_{2}=2 e_{1}, K e_{3}=6 e_{2}
$$


Let $\left\{f_{i}\right\}_{i=1}^{3}=\left\{2 e_{1}, 2 e_{1}, 6 e_{2}\right\}$, we know $\left\{f_{i}\right\}_{i=1}^{3}$ is a $K$-Frame for $H$ by Lemma 4.2. We can take $K^{+}$as follows

$$
K^{+} e_{1}=\frac{e_{1}+e_{2}}{4}, K^{+} e_{2}=\frac{e_{3}}{6}, K^{+} e_{3}=0 .
$$

It's easy the calculate the adjoint of $K$ as follows

$$
K^{+} e_{1}=2 e_{1}+2 e_{2}, K^{+} e_{2}=6 e_{3}, K^{+} e_{3}=0 .
$$

Since $\left\{f_{i}\right\}_{i=1}^{3}$ is a $K$-frame for $H$, by the definition of $K$-frame we can get $0<A \leq 1$, so let me take $A=1$. From (5.1) we can obtain $\left\|K^{+}\right\|=\frac{1}{2 \sqrt{2}}$. Let $\alpha=0.95, \beta=0.96, \gamma=0.001$, it's easy the check that $\max \left\{\alpha+\gamma \sqrt{A^{-1}}\left\|K^{+}\right\|, \beta\right\}<1$ holds. Now, take $g_{1}=e_{1}, g_{2}=e_{1}, g_{3}=5 e_{2}$, for any $c_{i}(i=1,2,3)$, we have

$$
\begin{aligned}
& \left\|\sum_{k=1}^{3} c_{k}\left(f_{k}-g_{k}\right)\right\|=\sqrt{\left(c_{1}+c_{2}\right)^{2}+c_{3}^{2}}, \\
& \gamma\left(\sum_{i=1}^{n} c_{k}^{2}\right)^{\frac{1}{2}}=0.001 \sqrt{c_{1}^{2}+c_{2}^{2}+c_{3}^{2}} \geq 0, \\
& \alpha\left\|\sum_{k=1}^{n} c_{k} f_{k}\right\|=0.95 \sqrt{4\left(c_{1}+c_{2}\right)^{2}+36 c_{3}^{2}}=\sqrt{3.16\left(c_{1}+c_{2}\right)^{2}+32.49 c_{3}^{2}}, \\
& \left\|\sum_{k=1}^{n} c_{k} g_{k}\right\|=\sqrt{3.6864\left(c_{1}+c_{2}\right)^{2}+23.04 c_{3}^{2}} \geq 0 .
\end{aligned}
$$

It is trivial to check that (4.1) holds. Moreover, we can show that $\left\{g_{i}\right\}_{i=1}^{3}=\left\{e_{1}, e_{1}, 5 e_{2}\right\}$ is a $P_{Q(R(K))} K-$ Frame for $H$. In fact, by the definitions of $T, U$ we get $Q f=U T^{*} f=\sum_{k=1}^{3}\left\langle f, f_{k}\right\rangle g_{k}, f \in H$. For any $f \in H$, suppose that $f=c_{1} e_{1}+c_{2} e_{2}+c_{3} e_{3}$, then

$$
Q f=\sum_{k=1}^{3}\left\langle c_{1} e_{1}+c_{2} e_{2}+c_{3} e_{3}, f_{k}\right\rangle g_{k}=4 c_{1} e_{1}+30 c_{2} e_{2},
$$

which implies that $R(Q)=\overline{\operatorname{span}}\left\{e_{1}, e_{2}\right\}$. By the definition of $K$ we can calculate $\|K\|=6$, now we leave the reader to verify that (4.3) holds.

\section{Conclusion}

In this article, controlled $K$-frames is first defined. Then, we examined the conditions that controlled $K$-frames are equivalent to $K$-frames (under certain conditions). At the end, the stability of the controlled $K$-Bessel sequences were checked under perturbation.

\section{REFERENCES}

[1] P. Balazs, J. P. Antoine, A. Grybos, Wighted and Controlled Frames, Int. J. Wavelets Multiresolut. Inf. Process. 8(1) (2010) 109-132.

[2] I. Bogdanova, P. Vandergheynst, J .P. Antoine, L. Jacques, M. Morvidone, Stereographic wavelet frames on the sphere, Appl. Comput. Harmon. Anal. (19) (2005) 223-252. 
[3] H. Bolcskei, F. Hlawatsch , H. G. Feichtinger,Frame theoretic analysis of over- sampled filter banks, IEEE Trans. Signal Process. 46 (1998) 3256-3268.

[4] P. Casazza, O. Christensen, Perturbation of operators and applications to frame theory, J. Fourier Anal. Appl. 3 (1997) 543-557.

[5] P. G. Casazza, G. Kutyniok, Frames of subspaces. Wavelets, frames and operator theory, College Park, MD, Contemp. Math., vol.345. American Mathematical Society, Providence,(2004) 87-113.

[6] P.G. Casazza, G. Li .S. Kutyniok, Fusion frames and distributed processing, Appl. Comput. Harmon. Anal.25 (2008) $114-132$.

[7] O. Christensen, An introduction to frames and Riesz bases, Birkhäuser, Boston 2003.

[8] N. E. Duday Ward, J. R. Partington, A construction of rational wavelets and frames in Hardy-Sobolev space with applications to system modelling. SIAM.J.Control Optim.36 (1998) 654-679.

[9] R.J. Duffin, A.C. Schaeffer, A class of nonharmonic Fourier series, Trans. Math. Soc.72 (1952) 341-366.

[10] I. Daubechies, A. Grossmann, Y. Meyer, Painless non orthogonal expansions, J. Math. Phys. 27(1986) 1271-1283.

[11] Y. C. Eldar, Sampling with arbitrary sampling and reconstruction spaces and oblique dual frame vectors, J. Fourier. Anal. Appl. 9(1) (2003) 77-96.

[12] Y. C. Eldar, T. Werther, General framework for consistent sampling in Hilbert spaces, Int. J. Walvelets Multi. Inf. Process. $3(3)(2005) 347-359$.

[13] P. J. S. G. Ferreira, Mathematics for multimedia signal processing II: Discrete finite frames and signal reconstruction, In: Byrnes, J.s. (ed.) signals processing for multimedia, PP. 35-54.IOS press, Amsterdam (1999).

[14] D. Hua and Y. Huang, Controlled K-g-frames in Hilbert spaces, Results. Math. (2016) DOI 10.1007/s00025-016-0613-0.

[15] A. Khosravi and K. Musazadeh, Controlled fusion frames, Methods Funct. Anal. Topol. 18(3) (2012), 256-265.

[16] K. Musazadeh and H. Khandani, Some results on controlled frames in Hilbert spaces, Acta Math. Sci. 36B(3) (2016), $655-665$.

[17] A. Rahimi, A. Fereydooni, Controlled G-Frames and Their G-Multipliers in Hilbert spaces , An. tiin. Univ. Ovidius Constana, 2(12) (2013), 223-236.

[18] M. Rashidi-Kouchi and A. Rahimi, On controlled frames in Hilbert $C^{*}$-modules, Int. J. Wavelets Multiresolut. Inf. Process. 15(4) (2017), Art. ID 1750038.

[19] T. Strohmer, R. Jr. Heath, Grass manian frames with applications to coding and communications, Appl. Comput. Harmon. Anal. 14 (2003), 257- 275.

[20] W. Sun, G-frames and g-Riesz bases, J. Math. Anal. 322 (2006) 437-452.

[21] X. Xiao, Y. Zhu, L. Găvruta, Some Properties of K-Frames in Hilbert Spaces , Results. Math. 63 (2013), $1243-1255$. 